\title{
Implication of Electroweak Monopole
}

\author{
Y. M. Cho',2 \\ ${ }^{1}$ Center for Quantum Spacetime, Sogang University, Seoul 04107, Korea \\ ${ }^{2}$ School of Physics and Astronomy, Seoul National University, Seoul 08826, Korea
}

\section{Abstract}

The electroweak monopole in the standard model and it's physical implications are discussed. It could generate the hitherto unknown magnetic current which has unlimited practical applications. Moreover, in cosmology it could generate the primordial magnetic black holes which might explain the dark matter, become the seed of the large scale structures of the universe, and be the source of the intergalactic magnetic field. Most importantly, if detected, it becomes the first magnetically charged and stable topological elementary particle in the history of physics.

Keywords: electroweak monopole, Cho-Maison monopole, monopole production in early universe DOI: 10.31526/ACP.NDM-2020.24

Ever since Dirac [1] has introduced the concept of the magnetic monopole in 1931, the monopole has become an obsession in theoretical as well as experimental physics. The Dirac monopole has been generalized to the Wu-Yang monopole in QCD [2], 't Hooft-Polyakov monopole in Georgi-Glashow model [3], and the Dokos-Tomaras monopole in the grand unified theory [4].

In the interesting case of the electroweak theory of Weinberg and Salam, however, it has been asserted that there exists no topological monopole of physical interest [5]. The basis for this "non-existence theorem" is that with the spontaneous symmetry breaking the quotient space $S U(2) \times U(1) / U(1)_{\text {em }}$ allows no non-trivial second homotopy. This has led people to conclude that there is no topological structure in Weinberg-Salam model which can accommodate the monopole. However, in 1997 Cho and Maison have established that Weinberg-Salam model has a different type of monopole topology, and demonstrated the existence of a new type of monopole solution in the standard model $[6,7]$.

This was based on the observation that the Weinberg-Salam model, with the hypercharge $U(1)$, could be viewed as a gauged $C P^{1}$ model in which the (normalized) Higgs doublet plays the role of the $C P^{1}$ field. So the $S U(2)$ part of Weinberg-Salam model has exactly the same non-Abelian monopole topology $\pi_{2}\left(S^{2}\right)$ as the Georgi-Glashow model. Moreover, in the standard model the $U(1)_{Y}$ becomes non-trivial and thus has the Abelian monopole topology. So the standard model has the hybrid monopole topology which allows the electroweak monopole, a hybrid of 't Hooft-Polyakov and Dirac monopoles.

Originally the solution of Cho and Maison was obtained by a numerical integration [6]. But the mathematically rigorous existence proof has since been established, and the solutions are referred to as Cho-Maison monopole [7].

Although the electroweak monopole is the electroweak generalization of the Dirac monopole, it is different from the Dirac monopole $[8,9,10]$. First, the Dirac monopole does not have to exist in electrodynamics. It exists only when the $U(1)$ bundle becomes non-trivial. But the electroweak monopole must exist if the standard model is correct, because the standard model has the monopole topology. Second, the magnetic charge is twice that of the Dirac monopole. This is because the period of the $U(1)$ subgroup of $S U(2)$ is $4 \pi$. This makes the period of the $U(1)_{(\mathrm{em})}$ of the electroweak theory to $4 \pi$, while the period of the $U(1)$ in electrodynamics is $2 \pi$. This doubles the magnetic charge of the electroweak monopole. So we can clearly distinguish the electroweak monopole from the Dirac monopole.

As we have pointed out, the electroweak monopole should exist if the standard model is correct. So the final test of the standard model should come from the discovery of the electroweak monopole, not the Higgs particle [6, 7]. In fact, the discovery of this monopole must be viewed as the topological test of the standard model.

The experimental detection of Dirac monopole has been a blind search in the dark room because we could not predict the mass [11]. Although the Cho-Maison monopole is singular and has an infinite energy, we can estimate the mass to be of the order of 4 to $10 \mathrm{TeV}[8,9,10]$. This is because the monopole acquires the mass from the same Higgs mechanism which gives the mass to the W-boson, except that the monopole has the magnetic coupling which makes the mass roughly hundred times heavier than the $\mathrm{W}$-boson mass. Moreover, one can regularize the monopole and argue that the regularized monopole mass may not be larger than $5.5 \mathrm{TeV}$ but has the BPS lower bound $2.98 \mathrm{TeV}$, more probably $3.75 \mathrm{TeV}[12,13,14]$.

The experimental confirmation of the electroweak monopole has become one of the most urgent issues after the discovery of the Higgs particle at LHC, so that ATLAS and MoEDAL detectors at LHC are actively searching for the monopole [15, 16, 17]. And the recent MoEDAL result already excludes the monopole with mass below $3 \mathrm{TeV}$. On the other hand, they may have no chance 

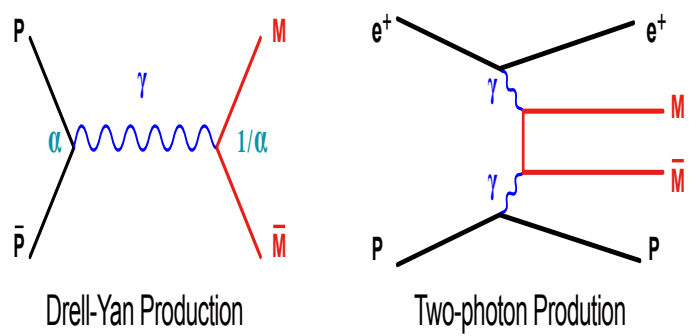

FIGURE 1: The Feynman diagrams of the popular monopole production mechanism given by Drell-Yan process and two-photon fusion process.

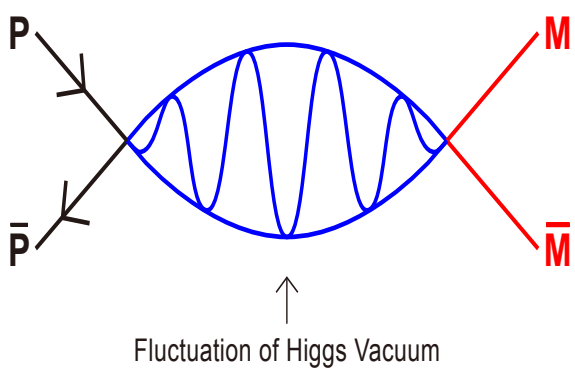

FIGURE 2: The topological monopole production mechanism induced by the thermal fluctuation of the Higgs vacuum.

to detect it if the mass becomes larger than $7 \mathrm{TeV}$, because the present $14 \mathrm{TeV}$ LHC can produce the monopole pair only when the mass is smaller than $7 \mathrm{TeV}$. In this case we may have to search for the remnant monopoles in the present universe which have been produced in the early universe, or else wait for the next LHC energy upgrading. So it is encouraging that IceCube and similar detectors are aiming to detect the remnant monopoles [18, 19, 20].

To detect the remnant monopoles, we have to understand the production mechanism in the early universe to estimate the monopole density at present universe. The monopole production mechanism in early universe is believed to depend on the type of phase transition [21, 22]. However, what is important for the monopole production is the change of topology induced by thermal fluctuation of the Higgs vacuum, which becomes insignificant when the temperature cools down below the Ginzburg temperature [23]. This implies that the electroweak monopole production starts during the electroweak phase transition around $100 \mathrm{GeV}$ and stops after the universe cools down below the Ginzburg temperature around $60 \mathrm{GeV} \mathrm{[10].}$

Although the electroweak monopoles are produced copiously around the Ginzburg temperature, they are annihilated as soon as created. And this annihilation process continues very long time, till the universe cools down to around $30 \mathrm{MeV}$, when the monopole-antimonopole capture radius becomes equal to the free streaming distance. This is basically because the attraction between monopole and anti-monopole is much bigger, $1 / \alpha$-times bigger than the electromagnetic interaction. As a result the density of the electroweak monopoles at present universe turns out to be very small, about $10^{-11}$ of the critical density. This assures that, unlike the grand unification monopole, the electroweak monopole has no monopole problem in cosmology. Nevertheless there are enough remaining monopoles in the present universe that we could detect without much difficulty [10].

As importantly, they play very important roles in cosmology. As the only magnetically charged heavy and stable particle in the early universe, it becomes an ideal source of density perturbation in the radiation dominant era. So, it could easily become the seed of the primordial black holes and the large scale structures of the universe.

There is experimental evidence which supports this. The recent observation of the strong radial magnetic field near the galactic center strongly indicates that it could have been generated by the electroweak monopoles buried in the galactic center [24]. This suggests that the monopoles could have been the seed of the large scale structure of the universe.

Moreover, the primordial black holes generated by the electroweak monopoles could explain the dark matter. Although the electroweak monopoles by themselves cannot account for the dark matter, the primordial black holes created by the monopoles could easily acquire huge mass and account for the dark matter. The primordial black hole has been proposed to be a candidate of dark matter for a long time [25]. But there have been few compelling mechanism how the primordial black holes could be created. Our analysis strongly indicates that the electroweak monopole could naturally create them.

The electroweak monopole has another important implication in cosmology. It could become the source of the intergalactic magnetic field. There has been no good explanation of the origin of the intergalactic magnetic field. The magnetic field of the remnant monopoles could explain this. Moreover, as the heaviest relativistic magnetically charged particles in the universe, they become a natural source of ultra-high energy cosmic rays. 
Our result provides a useful tip for the remnant monopole detection experiments. Certainly there are enough remnant electroweak monopoles to be detected. But when they pass through the earth atmospheric sphere, they loose most of the kinetic energy and slow down quickly when they reach the earth surface. So most of them are expected to be trapped in the earth surface [10].

This tells the followings. First, it would be extremely difficult for the monopoles to arrive at detectors buried more than $2 \mathrm{~km}$ under the iceberg at the south pole or under the Mediterranean sea. Second, a best way to detect the remnant monopole is to locate the detector at high altitude. The remnant monopole detection experiments should keep these points in mind.

Moreover, our analysis provides an important information for the MoEDAL and ATLAS monopole detection experiments at LHC $[15,16,17]$. Our result suggests that the monopole production mechanism at LHC should be the topological production after the electroweak phase transition, which is induced by the thermal fluctuation of the Higgs vacuum. This suggests that the radius of the fireball at LHC must be bigger than the correlation length of the Higgs vacuum, and must last long enough for the Higgs vacuum to fluctuate.

More importantly, our result strongly implies that the popular monopole production mechanism at LHC that the monopoles are produced in pairs by Drell-Yan process and/or two-photon fusion process in doubt [26]. To see this we compare the popular Drell-Yan and two-photon processes shown in Fig. 1 and our topological monopole production mechanism shown in Fig. 2. The contrast between the two figures is unmistakable. The photon plays the crucial role in Fig. 1, but Fig. 2 involves no photon and can not be described by any Feynman diagram. So it is very important for us to determine which is the correct monopole production mechanism.

In principle we can determine which is the correct production mechanism by experiment. The notable difference between the two mechanisms is the coupling strength. In the Drell-Yan and two-photon production the coupling is uniquely fixed by the fine structure constant, but in the topological production this is not so. Here the coupling is not given by any known fundamental constant. This means that the monopole production probability in two mechanisms should be different. So, by measuring the monopole production probability at LHC, we could tell which is correct.

\section{ACKNOWLEDGEMENTS}

The work is supported in part by the National Research Foundation funded by the Ministry of Education (Grant 2018-R1D1A1B07045163) and by Center for Quantum Spacetime, Sogang University, Korea.

\section{References}

[1] P.A.M. Dirac, Proc. Roy. Soc. London, A133, 60 (1931); Phys. Rev. 74, 817 (1948).

[2] T.T. Wu and C.N. Yang, Phys. Rev. D12, 3845 (1975); Y.M. Cho, Phys. Rev. Lett. 44, 1115 (1980); Phys. Lett. B115, 125 (1982).

[3] G. 't Hooft, Nucl. Phys. B79, 276 (1974); A.M. Polyakov, JETP Lett. 20, 194 (1974); M. Prasad and C. Sommerfield, Phys. Rev. Lett. 35, 760 (1975).

[4] C. Dokos and T. Tomaras, Phys. Rev. D21, 2940 (1980).

[5] S. Coleman, Aspects of Symmetry (Cambridge Univ. Press, 1985); T. Vachaspati and M. Barriola, Phys. Rev. Lett. 69, 1867 (1992); M. Barriola, T. Vachaspati, and M. Bucher, Phys. Rev. D50, 2819 (1994).

[6] Y.M. Cho and D. Maison, Phys. Lett. B391, 360 (1997); W.S. Bae and Y.M. Cho, JKPS 46, 791 (2005).

[7] Yisong Yang, Proc. Roy. Soc. London, A454, 155 (1998); Yisong Yang, Solitons in Field Theory and Nonlinear Analysis (Springer Monographs in Mathematics), p. 322 (Springer-Verlag) 2001.

[8] Kyoungtae Kimm, J.H. Yoon, and Y.M. Cho, Eur. Phys. J. C75, 67 (2015).

[9] Y.M. Cho, Kyoungtae Kimm, J.H. Yoon, Phys. Lett. B761, 203 (2016).

[10] Y.M. Cho, Phil. Trans. R. Soc. A377, 0038 (2019).

[11] B. Cabrera, Phys. Rev. Lett. 48, 1378 (1982).

[12] J. Ellis, N.E. Mavromatos, and T. You, Phys. Lett B756, 29, (2016).

[13] F. Blaschke and P. Benes, Prog. Theor. Exp. Phys. 073B03 (2018).

[14] Pengming Zhang, Liping Zou, and Y. M. Cho, Eur. Phys. J. C80, 280 (2020).

[15] B. Acharya et al. (MoEDAL Collaboration), Phys. Rev. Lett. 118, 061801 (2017); Phys. Rev. Lett. 123, 021802 (2019).

[16] B. Acharya et al. (MoEDAL Collaboration), arXiv:2002.00861 [hep-ex], Jan 2020, submitted to Phys. Rev. Lett.

[17] ATLAS Collaboration, Phys. Rev. Lett. 109, 261803 (2012); Euro. Phys. J. C75, 362 (2015); Phys. Rev. Lett. 124, 031802 (2020).

[18] R. Abbasi et al. (IceCube Collaboration) Phys. Rev. D87, 022001 (2013); M. Aartsen et al. (IceCube Collaboration), Eur. Phys. J. C74, 2938 (2014).

[19] S. Adrián-Martinez et al. (ANTARES Collaboration), Astropart. Phys. 35, 634 (2012).

[20] A. Aab et al. (Pierre Auger Collaboration) Phys. Rev. D94, 082002 (2016).

[21] T.W.B. Kibble, J. Phys. A9 1387 (1976).

[22] J.P. Preskill, Phys. Rev. Lett. 43, 1365 (1979).

[23] V. Ginzburg, Sov. Phys. Solid State 2, 1824 (1960).

[24] R. Eatough et al., Nature 591, 391 (2013); M. Zamaninasab, E. Clausen-Brown, T. Savolainen, A. Tchekhovskoy, Nature 510, 126 (2014).

[25] G. Chapline, Nature 253, 251 (1975); P. Frampton, M. Kawasaki, F. Takahashi, and T. Yanagida, JCAP 04, 023 (2010); B. Carr, F. Kuhnel, M. Sandstad, Phys. Rev. D94, 083504 (2016).

[26] T. Dougall and S. Wick, Euro. Phys. J. A39, 213 (2009); L. Epele, H. Fanchiotti, C. Cannl, V. Mitsou, and V. Vento, Euro. Phys. J. Plus 127, 60 (2012). 